J Urol Nephrol

June 2015 Vol.:2, Issue:1

(C) All rights are reserved by Vijayendra et al

\section{Pelvic and Abdominal Lipomatosis - A Rare Case Report with Atypical Presentations}

Keywords: Pelvic lipomatosis; LUTS; Graveluria; Obstructive uropathy; Pear shaped bladder; Tear drop sign; Cystitis cystic a

\section{Abstract}

Pelvic lipomatosis is defined as a condition characterized by the diffuse proliferation of nomal mature fatty tissue in the perirecta and perivesical region. We present a rare case of 50 years male, nomotensive, nomoglycemic and low BMI with progressive LUTS (lower uninary tract symptoms) over the last 10 years and episodes of ureteric colic spontaneously relieved by stone passage. The diagnosis of pelvic lipomatosis was established on the basis of clinic al and radiological findings. The patient was managed with bilateral PCN which was changed periodically and patient achieved a nadir creatinine to $2.9 \mathrm{mg} / \mathrm{dl}$ from $5.4 \mathrm{mg} / \mathrm{dl}$. Cystoscopy and biopsy were consistent with Cystitis Cystica.

\section{Introduction}

Pelvic lipomatosis (PL) is a disease of unknown cause, characterized by overgrowth of mature, nonmalignant fat cells in the pelvic region, especially in perivesical and perirectal spaces and absence of delimitation by a capsule. It is a rare disease; the incidence of PL in the United States was estimated as 0.6 to 1.7 per 100,000 . Pelvic lipomatosis is known to be more prevalent in males and African Americans. The clinical presentation of PL is variable and related to the compressive phenomenon on the urinary system (increased frequency, dysuria, nocturia, and hematuria), lower intestinal tract

\section{Journal of}

Urology \& Nephrology

\author{
Vijayendra SK ${ }^{1 *}$, Bernard $\mathrm{A}^{1}$, Kapoor $\mathrm{D}^{2}$ \\ Khumukcham $\mathbf{S}^{1}$, Singh $\mathrm{AK}^{1}$ and Sinam RS ${ }^{1}$ \\ ${ }^{1}$ Department of Urology, Regional Institute of Medical Sciences, \\ Imphal, India \\ ${ }^{2}$ Senoir Resident, Dr. Baba Saheb Ambedkar Hospital, New Delhi, \\ India \\ Address for Correspondence \\ Vijayendra S. Kanwar, Department of Urology, Regional Institute of \\ Medical Sciences, Imphal, India, E-mail: vijayendrakanwar@gmail.com \\ Submission: 25 May 2015 \\ Accepted: 23 June 2015 \\ Published: 29 June 2015 \\ Reviewed \& Approved by: Dr. Ahmed El-Zawahry, Assistant \\ Professor, Department of Surgery, Southern Illinois University, \\ Carbondale, USA
}

(constipation, tenesmus, rectal bleeding, and ribbon-like stools with mucus), and vascular system (edema of lower extremities) [1,2].

\section{Case History}

A 50-years-old male presented to us with history of pain in the left flank which got relieved by spontaneously passage of stones and progressive LUTS for the last 10 years. He reported history of graveluria thrice in last one year. His serum creatinine level was 5.4 $\mathrm{mg} / \mathrm{dl}$. An ultrasound revealed bilateral gross hydroureteronephrosis, and a thick-walled bladder with normal prostate. Nephrostogram showed bilateral hydroureteronephrosis (HDUN) with tortuous and dilated ureters and bladder placed at a higher position with extrinsic compression (Figure 1). CT revealed marked fat densities in the pelvis and abdomen, encasing the bladder and rectum with a thick wall,

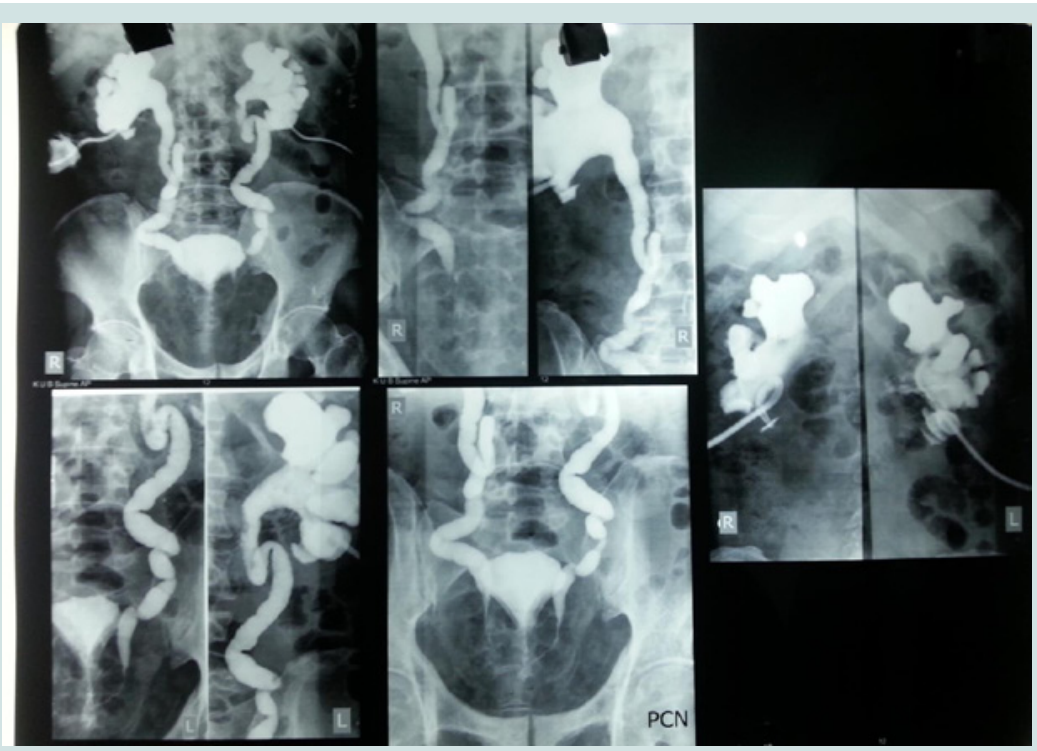

Figure 1: IVU showing B/L gross HDUN with small pulled up externally compressed bladder giving "tear drop/ pear sign". 
Citation: Vijayendra SK, Bernard A, Kapoor D, Khumukcham S, Singh AK, et al. Pelvic and Abdominal Lipomatosis - A Rare Case Report with Atypical Presentations. J Urol Nephrol. 2015;2(1): 3.

resulting in a bladder with a small capacity (Tear drop sign/ pear/ gourd-shaped bladder) suggestive of lipomatosis (Figure 2). Ureteral stenting was not possible because of high bladder neck, multiple papillary projections and non visualization of bilateral ureteric orifice hence Bilateral percutaneous nephrolithotomy (PCN) was done. Serum Creatinine started decreasing to $2.9 \mathrm{mg} / \mathrm{dl}$ in one month. The patient general condition improved. CPE (cysto-prostatoscopic uretheral examination) showed multiple papillary fronds shaped growth and elongated prostatic urethra (Figure 3), biopsy came as Cystitis Cystica. Patient was offered option of augumentation cystoplasty and ureteric reimplant but he preferred to remain on bilateral PCN and surveillance. A second CPE and biopsy was done at one year interval with HPR as Cystitis Cystica. His serum creatinine is stable at $3 \mathrm{mg} / \mathrm{dl}$ for the last 15 months. As for now patient is on regular follow-up.

\section{Discussion}

The etiology of Pelvic lipomatosis is unknown and it may remain dormant for years [3], but it is suggested that obesity plays a role, and it has been found in half of the patients afflicted with obesity. Approximately half of the patients present with lower urinary tract symptoms, and about a quarter present with bowel complaints.

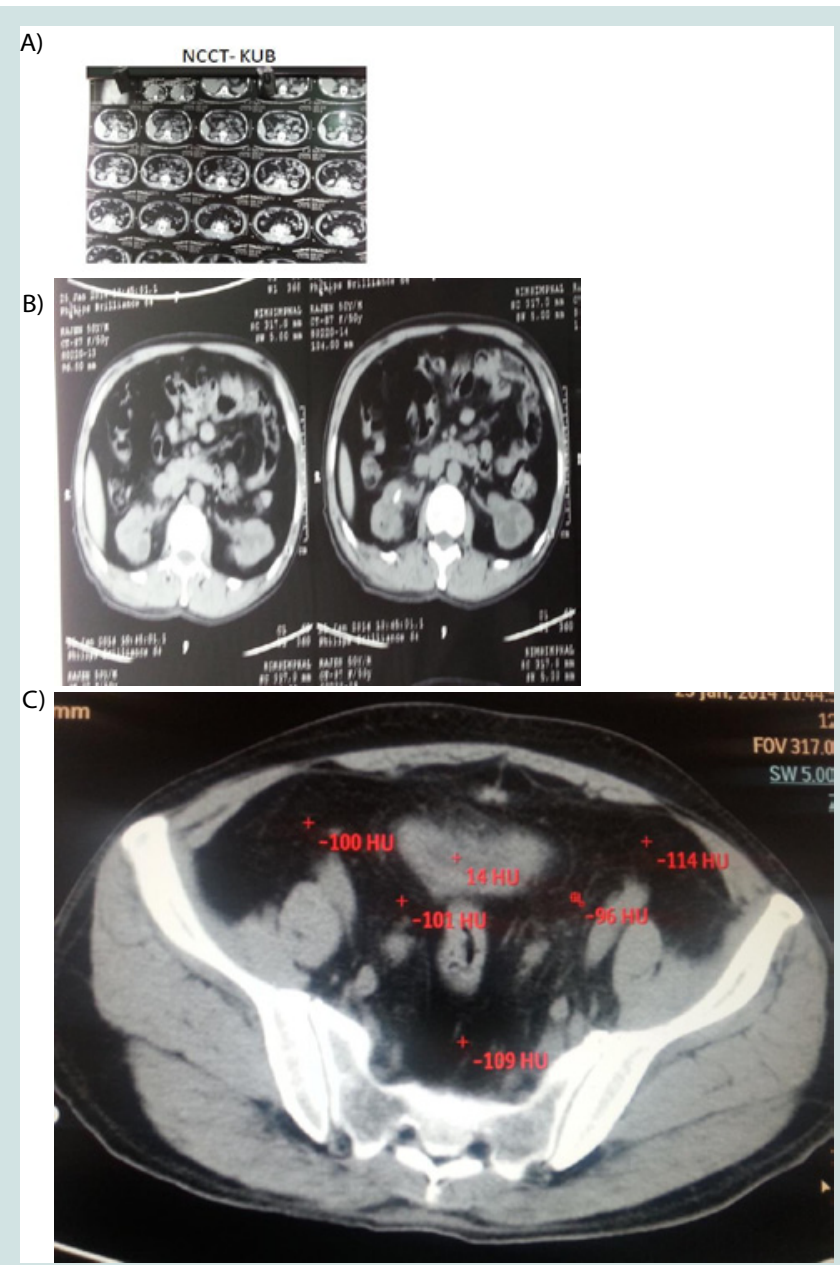

Figure 2: NCCT KUB depicting fat $\mathrm{HU}(-80$ to -120$)$ in abdomen and pelvis consistant with pelvic Lipomatosis.

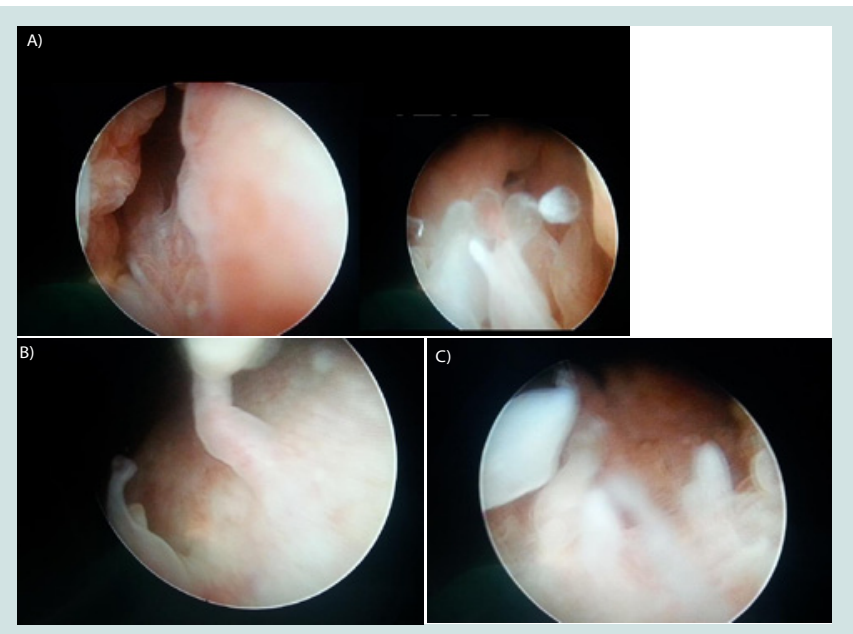

Figure 3: Cystoscopy with typical frond like projections.

Suprapubic pain or lumps, backache, flank pain, or perineal discomfort may be other clinical manifestations. Hypertension is common, occurring in 35 to $74 \%$ of patients. Pelvic Lipomatosis is more common in blacks and diabetics. Hydronephrosis develops in at least $1 / 3$ of the patients evaluated. It often presents with bilateral hydronephrosis in 19\% of patients. The cause of hydronephrosis is distal encasement of the ureter by lipomatosis lesions. Renal failure occurs in $6 \%$ of all reported cases [1,2]. However our patient did not have the classical clinical symptoms and sign, the patient was normotensive, normoglycemic, with a low BMI and presented with ureteric colic and spontaneous graveluria superimposed on chronic LUTS and did not have any bowel symptoms in although the CT (computed tomography) was suggestive of abdominal lipamatosis .

Computed tomography (CT) is the cornerstone of the diagnosis of pelvic lipomatosis, as the absorption co-efficiency of pelvic fatty tissue calculated by computer is distinct from that of other pelvic structures in the CT scan. On pelvic and abdominal CT (computed tomography), the bladder and rectosigmoid are surrounded and displaced by homogenous tissue with low attenuation ( -40 to -100 $\mathrm{HU}$ ), signifying fat content. An MRI can also be used for diagnosis, as it permits the characterization of fat planes, and it provides the delineation of an elevated bladder base and the elongation of a posterior urethra. Cystoscopy detects abnormalities in $75 \%$ of patients and most often includes cystitis glandularis and cystitis cystic. These proliferative changes may occur secondary to obstruction of bladder wall drainage, leading to an altered environment rich in protein fluid, which is a nutritional medium for proliferation of tissue [1] and both of these lesions have been regarded as a premalignant lesion of adenocarcinoma of the bladder [2].

There is no specific treatment recommendation for pelvic lipomatosis and the treatment options vary from long-term antibiotics, transurethral resection of the lesions to cystectomy and urinary diversion [4] in the form of ileal conduit, nephrostomy tube or vesicostomy, to relieve urinary obstruction associated with pelvic lipomatosis [5]. Steroids, antibiotics, dietary control and radiation therapy have been tried without any success. Radiological evaluation and serum creatinine estimation should be regularly performed and it would be reasonable that patients undergo annual or biannual 
Citation: Vijayendra SK, Bernard A, Kapoor D, Khumukcham S, Singh AK, et al. Pelvic and Abdominal Lipomatosis - A Rare Case Report with Atypical Presentations. J Urol Nephrol. 2015;2(1): 3.

\section{ISSN: $2380-0585$}

cystoscopies unless storage symptoms or hematuria develop which would require more frequent examinations $[2,6]$. Long term follow up is essential due to the risk for adenocarcinoma bladder.

\section{References}

1. Heyns CF, De Kock ML, Kirsten PH, van Velden DJ (1991) Pelvic lipomatosis associated with cystitis glandularis and adenocarcinoma of bladder. $\mathrm{J}$ Uro 145: 364-366.

2. Sözen S, Gürocak S, Uzüm N, Biri H, Memiş L (2004) The importance of re-evaluation in patients with cystitis glandularis associated with pelvic lipomatosis: a case report. Urol Oncol 22: 428-430.
3. Klein FA, Smith MJ, Kasenetz L (1988) Pelvic lipomatosis: 35-year experience. J Urol 139: 998-1001.

4. Michajłowski J, Matuszewski M, Kłącz J, Gibas A, Biernat W, et al. (2011) Acute urinary retention in a patient with extended cystitis glandularis. Cent European J Urol 64: 94-96.

5. Halachmi S, Moskovitz B, Calderon N, Nativ O (1996) The use of an ultrasonic assisted lipectomy device for the treatment of obstructive pelvic lipomatosis. Urology 48: 128-130.

6. Hudolin T, Kastelan Z, Goluza E, Basić-Jukić N, Sosić H (2010) Pelvic and retroperitoneal lipomatosis: case report. Acta Clin Croat 49: 465-468. 